\title{
A fifth-order finite volume weighted compact scheme for solving one-dimensional Burgers' equation
}

\author{
Yan $\mathrm{Guo}^{\mathrm{a}}$, Yu-feng Shi ${ }^{\mathrm{b}, *}$, Yi-min $\mathrm{Li}^{\mathrm{b}}$ \\ ${ }^{a}$ College of Sciences, China University of Mining and Technology, XuZhou 221116, \\ China. \\ ${ }^{b}$ School of Electric Power Engineering, China University of Mining and Technology, \\ XuZhou 221116, China.
}

\begin{abstract}
In the present paper, a high-order finite volume compact scheme is proposed to solve one dimensional Burgers' equation. The nonlinear advective terms are computed by the fifth-order finite volume weighted upwind compact scheme, in which the nonlinear Weighted Essentially Non-Oscillatory weights are coupled with lower order compact stencils. The diffusive terms are discretized by using the finite volume six-order Padé scheme. The strong stability preserving third-order Runge-Kutta time discretizations is used in this work. Numerical results are compared with the exact and some existing numerical solutions to demonstrate the essentially non-oscillatory and high resolution of the proposed method. The numerical results are shown to be more accurate than some numerical results given in the literature.
\end{abstract}

Keywords: Burgers' equation, finite volume method, compact schemes, weighted essentially non-oscillatory scheme, Padé schemes

\section{Introduction}

In this paper, the following one-dimensional Burgers' equation is considered

$$
\frac{\partial u}{\partial t}+u \frac{\partial u}{\partial x}=\nu \frac{\partial^{2} u}{\partial x^{2}}, x \in(a, b), t \in(0, T],
$$

\footnotetext{
*Corresponding author

Email addresses: yanguo@cumt.edu.cn (Yan Guo ), shiyufeng@cumt.edu.cn (Yu-feng Shi ), liyimin@cumt.edu.cn (Yi-min Li )
} 
4 with initial condition

$$
u(x, 0)=f(x), x \in[a, b],
$$

and boundary condition

$$
u(a, t)=\alpha, u(b, t)=\beta, t \in(0, T],
$$

$6 \quad$ where $\nu=\frac{1}{R e}$ and $R e$ called the Reynolds number.

The Burgers' equation is the simplest well-known nonlinear model equation from fluid dynamics. It is a simplified form of the Navier-Stokes equations that contain nonlinear advection and diffusion terms. The analytical solutions were obtained by using the Hopf-Cole transformation $[1,2]$. Benton and Platzman have surveyed the exact solutions of the one-dimensional Burgers' equation with different boundary and initial conditions in [3]. For some special initial and boundary conditions, the analytic solutions involve infinite series, which may converge very slowly for large Reynolds number.

In the past few years, the numerical solutions of (1) have attracted a lot of attention, and a variety of numerical methods have been proposed for this equation. A review of numerical methods for nonlinear Burgers' equation can be found in [4], such as finite difference method [5, 6, 7, 8, 9], B-spline method $[10,11]$ and cubic Hermite collocation method [12]. Jiwari and Mittal proposed lots of numerical methods for Burgers' equation, such as weighted average differential quadrature method [13], haar wavelet quasilinearization approach [14], hybrid numerical scheme [15], modified cubic B-splines collocation method [16], numerical scheme based on differential quadrature method [17] and differential quadrature method [18]. Recently, Mukundan proposed efficient numerical techniques for Burgers' equation [19] and Kumar proposed a composite numerical scheme for the numerical simulation of coupled Burgers' equation [20]. The present numerical results are also compared with some other numerical solutions, such as local discontinuous galerkin finite element method [21], automatic differentiation method [22], meshless method of lines [23] and polynomial based differential quadrature method[24].

In 1992, Lele developed high-order linear compact difference schemes based on implicit interpolations in [25]. These implicit schemes are very accurate in smooth regions, and have spectral-like resolution properties by using the global grid. However, these classical linear compact schemes necessarily produce non-physical oscillations when they are applied directly to flow with discontinuities. In recent years, there were a variety of methods to overcome this problem. Hybrid schemes which coupling a fifth-order compact 
upwind algorithm in conservation form with WENO (weighted essentially non-oscillatory) scheme [26] were proposed in [27] for shock-turbulence interaction. A new class of linear and hybrid weighted nonlinear schemes were recently proposed in $[28,29]$ based on the cell-centered compact scheme. Another methodology for capturing discontinuities has been proposed by Deng [30]. They developed nonlinear compact schemes, where adaptive compact stencils are used. Deng and Zhang [31] also proposed weighted compact nonlinear schemes (WCNS) based on the weighted technique. According to the idea of the WENO schemes, Jiang [32] proposed weighted compact schemes where the final compact scheme was a weighted combination of compact substencils. Ghosh employed the idea that is described in [32], and developed a class of upwind finite difference compact-reconstruction WENO schemes [33], where lower order compact stencils are identified at each interface and combined using the WENO weights. In recent years, a lot of papers dealing with compact schemes that were based on finite volume method can be found in the literature. Kobayashi proposed a class of Padé finite volume methods for linear equations in [34]. In [35], Piller proposed a high-order compact finite volume method for the scalar advection-diffusion equation, where advective fluxes are computed by the compact scheme introduced in [27] and the diffusive fluxes are discretized by using the Padé methods [34].

In this paper, a high-order finite volume compact scheme is proposed for solving Bugers' equation, where a fifth-order upwind weighted compactWENO scheme is used for the numerical approximation of advective terms, while the sixth-order Padé finite volume method proposed by Pirozzoli [27] is applied to approximate the diffusive terms. Comparing with classical noncompact WENO schemes, the proposed compact schemes have spectral-like resolution properties by using the global grid. In recent years, the compact finite difference schemes have been successfully used for solving Burgers' equation $[5,6,8,9]$. The classical linear central compact scheme for inviscid nonlinear Burgers' equation often yields oscillatory solutions across discontinuities. To achieve the essentially non-oscillatory properties of the present compact scheme, lower order compact stencils are combined with WENO weights to yield a fifth-order compact weighted interpolation. Then, the present scheme can maintain high order accuracy without oscillation. The accuracy of the proposed scheme will be checked by comparing the numerical against the exact solution. Comparisons of the numerical results with the exact solutions show that the proposed scheme is capable of achieving fifth-order accuracy. A number of tests are presented to show high resolution 
property of the proposed approach. Numerical comparisons are also made with some available results given in the literature, the numerical results show that the proposed method produces highly accurate results.

This paper is organized as follows. In Section 2, the present finite volume compact-WENO scheme is described. Comparisons of the numerical solutions with exact solutions and other numerical results are given in Section 3 , and the accuracy of the method is also investigated in this section. Some concluding remarks are given in Section 4.

\section{Finite volume compact-WENO schemes for Burgers' equation}

To construct the conservative finite volume scheme, we rewrite (1) as follows

$$
\frac{\partial u}{\partial t}+\frac{\partial f(u)}{\partial x}=\nu \frac{\partial^{2} u}{\partial x^{2}}, x \in[a, b], t \in(0, T],
$$

where $f(u)=\frac{u^{2}}{2}$. The computational domain $[a, b]$ can be divided into $N$ cells as follows:

$$
a=x_{\frac{1}{2}}<x_{\frac{3}{2}}<\cdots<x_{N+\frac{1}{2}}=b .
$$

We denote cells by $I_{j}=\left[x_{j-\frac{1}{2}}, x_{j+\frac{1}{2}}\right]$. For simplicity, we consider an uniform grid with $h=\frac{b-a}{N}$. In the finite volume approach, (1) is integrated over all the mesh cells. For cell $I_{j}$ with cell faces $x_{j-\frac{1}{2}}$ and $x_{j+\frac{1}{2}}$, we can obtain the following equation

$$
\frac{\partial}{\partial t} \int_{x_{j-\frac{1}{2}}}^{x_{j+\frac{1}{2}}} u d x+f\left(u\left(x_{j+\frac{1}{2}}, t\right)\right)-f\left(u\left(x_{j-\frac{1}{2}}, t\right)\right)=\nu\left(u_{x}\left(x_{j+\frac{1}{2}}, t\right)-u_{x}\left(x_{j-\frac{1}{2}}, t\right)\right) .
$$

The cell-averaged value of cell $j$ is denoted as $\bar{u}_{j}$, which can be defined as

$$
\bar{u}\left(x_{j}, t\right)=\frac{1}{h} \int_{x_{j-\frac{1}{2}}}^{x_{j+\frac{1}{2}}} u(x, t) d x .
$$

${ }_{91}$ We approximate (2) by the following scheme

$$
\frac{d \bar{u}_{j}(t)}{d t}=-\frac{1}{h}\left(\left(\hat{f}_{j+\frac{1}{2}}-\hat{f}_{j-\frac{1}{2}}\right)-\nu\left(\left(\hat{u}_{x}\right)_{j+\frac{1}{2}}-\left(\hat{u}_{x}\right)_{j-\frac{1}{2}}\right)\right),
$$

92 with a global Lax-Friedrichs flux defined by

$$
\hat{f}_{j+\frac{1}{2}}=h\left(u_{j+\frac{1}{2}}^{-}, u_{j+\frac{1}{2}}^{+}\right)=\frac{1}{2}\left[f\left(u_{j+\frac{1}{2}}^{+}\right)+f\left(u_{j+\frac{1}{2}}^{-}\right)-\alpha\left(u_{j+\frac{1}{2}}^{-}-u_{j+\frac{1}{2}}^{+}\right)\right],
$$




$$
\frac{3}{10} u_{j-\frac{1}{2}}^{-}+\frac{6}{10} u_{j+\frac{1}{2}}^{-}+\frac{1}{10} u_{j+\frac{3}{2}}^{-}=\frac{1}{30} \bar{u}_{j-1}+\frac{19}{30} \bar{u}_{j}+\frac{10}{30} \bar{u}_{j+1} .
$$

The small length scale compact scheme (5) is very accurate and keeps good resolutions in smooth regions, but non-physical oscillations are generated when they are applied directly to some physical problems with discontinuities. Unfortunately, the amplitude does not decrease if we refine the grid. In this work, we adopt the main idea of the WENO scheme to form the present compact-WENO scheme. It uses a nonlinear convex combination of all candidate stencils to reduce spurious non-physical oscillations. In order to capture the shock precisely and keep high-order accuracy and high resolution in the smooth area, we construct a fifth order finite volume compact-WENO scheme by using three third-order compact interpolations as candidates. We follow the idea that is described in [33] and rewrite (5) as a weighted combination of three third order compact substencils, they are

$$
\begin{aligned}
& \frac{2}{3} u_{j-\frac{1}{2}}^{-}+\frac{1}{3} u_{j+\frac{1}{2}}^{-}=\frac{1}{6}\left(\bar{u}_{j-1}+5 \bar{u}_{j}\right), \\
& \frac{1}{3} u_{j-\frac{1}{2}}^{-}+\frac{2}{3} u_{j+\frac{1}{2}}^{-}=\frac{1}{6}\left(5 \bar{u}_{j}+\bar{u}_{j+1}\right), \\
& \frac{2}{3} u_{j+\frac{1}{2}}^{-}+\frac{1}{3} u_{j+\frac{3}{2}}^{-}=\frac{1}{6}\left(\bar{u}_{j}+5 \bar{u}_{j+1}\right) .
\end{aligned}
$$

We multiply (6) by linear weights $c_{0}=\frac{2}{10}, c_{1}=\frac{5}{10}, c_{2}=\frac{3}{10}$ respectively to obain linear compact scheme (5).

The WENO methodology is adopted for solving equations with discontinuous solutions. Then, the optimal linear weights $\left\{c_{0}, c_{1}, c_{2}\right\}$ are replaced by nonlinear weights $\left\{\omega_{0}, \omega_{1}, \omega_{2}\right\}$. The fifth-order finite volume upwind compactWENO approximations for advective terms can be obtained [36, 37]. Given the cell averages $\bar{u}_{j}$ for each cell $I_{j}$, we obtain fifth-order compact approximations for $u_{j+\frac{1}{2}}^{-}$based on stencils $\left\{x_{j-1}, x_{j}, x_{j+1}\right\}$, resulting in an implicit 
system given by

$$
\begin{aligned}
& \frac{2 \omega_{0}+\omega_{1}}{3} u_{j-\frac{1}{2}}^{-}+\frac{\omega_{0}+2\left(\omega_{1}+\omega_{2}\right)}{3} u_{j+\frac{1}{2}}^{-}+\frac{1}{3} \omega_{2} u_{j+\frac{3}{2}}^{-} \\
= & \frac{1}{6} \omega_{0} \bar{u}_{j-1}+\frac{5\left(\omega_{0}+\omega_{1}\right)+\omega_{2}}{6} \bar{u}_{j}+\frac{\omega_{1}+5 \omega_{2}}{6} \bar{u}_{j+1},
\end{aligned}
$$

where nonlinear weights $\omega_{k}$ can be taken as $[38,39]$

$$
\omega_{k}=\frac{\alpha_{k}^{z}}{\sum_{l=0}^{2} \alpha_{l}^{z}}, \quad \alpha_{k}^{z}=c_{k}\left(1+\left(\frac{\tau_{5}}{\beta_{k}+\epsilon}\right)^{p}\right), \quad k=0,1,2,
$$

where $\tau_{5}=\left|\beta_{2}-\beta_{0}\right|$, which can improve the order of accuracy around the smooth extrema as compared to the classic WENO scheme [40]. We set $p=2$ and $\epsilon=10^{-13}$ in our numerical computation. The classical smooth indicators $\beta_{k}(k=0,1,2)$ in $[40]$ are given by

$$
\begin{aligned}
& \beta_{0}=\frac{13}{12}\left(\bar{u}_{j-2}-2 \bar{u}_{j-1}+\bar{u}_{j}\right)^{2}+\frac{1}{4}\left(\bar{u}_{j-2}-4 \bar{u}_{j-1}+3 \bar{u}_{j}\right)^{2}, \\
& \beta_{1}=\frac{13}{12}\left(\bar{u}_{j-1}-2 \bar{u}_{j}+\bar{u}_{j+1}\right)^{2}+\frac{1}{4}\left(\bar{u}_{j-1}-\bar{u}_{j+1}\right)^{2}, \\
& \beta_{2}=\frac{13}{12}\left(\bar{u}_{j}-2 \bar{u}_{j+1}+\bar{u}_{j+2}\right)^{2}+\frac{1}{4}\left(3 \bar{u}_{j}-4 \bar{u}_{j+1}+\bar{u}_{j+2}\right)^{2} .
\end{aligned}
$$

From (8), we can observe that the nonlinear weights depend on the solutions, then we need to solve a tridiagonal system of (7) to get $u_{j+\frac{1}{2}}^{-}$at each time step. $u_{j+\frac{1}{2}}^{+}$can be obtained similarly. For more details about the scheme, readers can see [37].

\subsection{Reconstruction for diffusive terms}

To obtain a high-order scheme for Burgers' equation in smooth regions, sixth-order Padé finite volume scheme introduced in [34] is used for the discretization of the diffusive fluxes. The implicit interpolation formula for interior nodes can be written as

$$
\left.\frac{2}{11} \frac{\partial u}{\partial x}\right|_{j-\frac{1}{2}}+\left.\frac{\partial u}{\partial x}\right|_{j+\frac{1}{2}}+\left.\frac{2}{11} \frac{\partial u}{\partial x}\right|_{j+\frac{3}{2}}=\frac{51}{44} \frac{\bar{u}_{j+1}-\bar{u}_{j}}{\Delta x}+\frac{3}{44} \frac{\bar{u}_{j+2}-\bar{u}_{j-1}}{\Delta x} .
$$

It can be seen that the high orders of accuracy with relatively small stencils can be achieved. 


\subsection{Boundary conditions}

For the high-order compact scheme, we need to set appropriate boundary closures for both advective and diffusive terms. For the discretization of the advective term near left boundary nodes, a fifth-order approximation formulas is used in the present work. The formulas can be written as

$$
\frac{1}{5} \tilde{u}_{j-\frac{1}{2}}+\frac{4}{5} \tilde{u}_{j+\frac{1}{2}}=\frac{37}{60} \bar{u}_{j}+\frac{29}{60} \bar{u}_{j+1}-\frac{7}{60} \bar{u}_{j+2}+\frac{1}{60} \bar{u}_{j+3} .
$$

For the diffusive terms at boundary nodes, approximation formulae for the first-order derivatives of non-periodic problems can be derived from one-sided schemes, the 6th-order von Neumann boundary [34] is used in this work. The derived formulae at right boundary point is

$$
u_{N+\frac{1}{2}}+\frac{27}{23} u_{N-\frac{1}{2}}=\frac{3 h}{23}\left(\left.\frac{\partial u}{\partial x}\right|_{N+\frac{1}{2}}-\left.3 \frac{\partial u}{\partial x}\right|_{N-\frac{1}{2}}\right)+\frac{325}{138} \bar{u}_{N}-\frac{13}{69} \bar{u}_{N-1}+\frac{1}{138} \bar{u}_{N-2} .
$$

The derived formulae at left boundary point can be obtained similarly.

\subsection{Temporal discretization}

We use a strong stability preserving (SSP) high order Runge-Kutta time discretizations in this work. The third-order SSP Runge-Kutta method is

$$
\begin{aligned}
& u^{(1)}=u^{n}+\Delta t L\left(u^{n}\right), \\
& u^{(2)}=\frac{3}{4} u^{n}+\frac{1}{4} u^{(1)}+\frac{1}{4} \Delta t L\left(u^{(1)}\right), \\
& u^{n+1}=\frac{1}{3} u^{n}+\frac{2}{3} u^{(2)}+\frac{2}{3} \Delta t L\left(u^{(2)}\right),
\end{aligned}
$$

where $L(u)$ is the spatial operator. For $(3)$, we set $L=-\frac{1}{\Delta x_{j}}\left(\left(\hat{f}_{j+\frac{1}{2}}-\hat{f}_{j-\frac{1}{2}}\right)-\right.$ $\left.\nu\left(\left(\hat{u_{x}}\right)_{i+\frac{1}{2}}-\left(\hat{u_{x}}\right)_{i-\frac{1}{2}}\right)\right)$ in our computation.

The algorithm for the present FVCW scheme solving (1) consists of the following steps:

1. We need to solve the tridiagonal system of (7) to get $u_{j+\frac{1}{2}}^{-}$by using nonlinear weights (8) at each time step.

2. (9) coupled with (11) are used to obtain the diffusive fluxes.

3. The global Lax-Friedrichs flux (4) can be obtained based on step 1.

4. The right side of (3) can be computed based on step 2 and step 3 .

5. The third-order SSP Runge-Kutta method (12) is used to get the value $u_{j}^{n+1}$. 


\subsection{Convergence and linear stability analysis}

We consider the linear stability properties of the third-order SSP RungeKutta method (12) when coupled with FVCW scheme to solve Burgers' equation (1) by means of the von Neumann analysis. For nonlinear equation (1), we linearize and freeze coefficients, then the equation (1) can be written as following

$$
\frac{\partial u}{\partial t}+\lambda \frac{\partial u}{\partial x}=\nu \frac{\partial^{2} u}{\partial x^{2}},
$$

where $\lambda=\max |u|$. We consider a uniform spatial mesh. The semidiscrete equation is

$$
\frac{d \bar{u}_{j}(t)}{d t}=-\lambda \frac{1}{h}\left(\hat{u}_{j+\frac{1}{2}}-\hat{u}_{j-\frac{1}{2}}\right)+\frac{1}{h} \nu\left(\left(\hat{u}_{x}\right)_{j+\frac{1}{2}}-\left(\hat{u}_{x}\right)_{j-\frac{1}{2}}\right) .
$$

Equation (14) can be cast in matrix form as

$$
\frac{d \overline{\mathbf{u}}}{d t}=-\lambda \frac{1}{h} \mathbf{C} \hat{\mathbf{u}}+\frac{1}{h} \nu \mathbf{C} \hat{\mathbf{u}_{\mathbf{x}}},
$$

where

$$
\overline{\mathbf{u}}=\left[\begin{array}{c}
\bar{u}_{1} \\
\bar{u}_{2} \\
\vdots \\
\bar{u}_{N}
\end{array}\right], \hat{\mathbf{u}}=\left[\begin{array}{c}
\hat{u}_{\frac{3}{2}} \\
\bar{u}_{\frac{5}{2}} \\
\vdots \\
\bar{u}_{N+\frac{1}{2}}
\end{array}\right], \hat{\mathbf{u}_{\mathbf{x}}}=\left[\begin{array}{c}
\hat{u}_{x_{\frac{3}{2}}} \\
\bar{u}_{x \frac{5}{2}} \\
\vdots \\
\overline{u_{x+\frac{1}{2}}}
\end{array}\right], \mathbf{C}=\left[\begin{array}{cccc}
1 & & & -1 \\
-1 & 1 & & \\
& \ddots & \ddots & \\
& & -1 & 1
\end{array}\right] .
$$

We consider the inviscid equation $\frac{d \overline{\mathbf{u}}}{d t}=-\lambda \frac{1}{h} \mathbf{C} \hat{\mathbf{u}}$. Equation (5) with periodic boundary can be written in matrix form as

$$
\mathbf{A} \hat{\mathbf{u}}=\mathbf{B} \overline{\mathbf{u}},
$$

where

$$
\mathbf{A}=\left[\begin{array}{cccccc}
\frac{6}{10} & \frac{1}{10} & & & & \frac{3}{10} \\
\frac{3}{10} & \frac{6}{10} & \frac{1}{10} & & & \\
& \frac{3}{10} & \frac{6}{10} & \frac{1}{10} & & \\
& & \ddots & \ddots & \ddots & \\
& & & \frac{3}{10} & \frac{6}{10} & \frac{1}{10} \\
\frac{1}{10} & & & & \frac{3}{10} & \frac{6}{10}
\end{array}\right], \mathbf{B}=\left[\begin{array}{cccccc}
\frac{19}{30} & \frac{1}{3} & & & & \frac{1}{30} \\
\frac{1}{30} & \frac{19}{30} & \frac{1}{3} & & & \\
& \frac{1}{30} & \frac{19}{30} & \frac{1}{3} & & \\
& & \ddots & \ddots & \ddots & \\
& & & \frac{1}{30} & \frac{19}{30} & \frac{1}{3} \\
\frac{1}{3} & & & & \frac{1}{30} & \frac{19}{30}
\end{array}\right]
$$


The inviscid equation can be written as

$$
\frac{d \overline{\mathbf{u}}}{d t}=-\lambda \frac{1}{h} \mathbf{C A}^{-1} \mathbf{B} \overline{\mathbf{u}}
$$

where $\mathbf{C}, \mathbf{A}$ and $\mathbf{B}$ are circulant tridiagonal matrices. The eigenvalues of a circulant tridiagonal matrix are [41] presented as

$$
\lambda^{(k)}=\beta+(\alpha+\gamma) \cos \left(\frac{2 k \pi}{M}\right)-\mathbf{i}(\alpha-\gamma) \sin \left(\frac{2 k \pi}{M}\right), k=0, \cdots, M-1,
$$

where $\alpha, \beta$ and $\gamma$ are the subdiagonal, diagonal and superdiagonal terms, respectively. The eigenvalues of $\mathbf{C}, \mathbf{A}$ and $\mathbf{B}$ are

$$
\begin{aligned}
& \lambda_{\mathbf{C}}^{(k)}=1-\exp ^{-2 \pi \mathbf{i} k / M}, \\
& \lambda_{\mathbf{A}}^{(k)}=\frac{6}{10}+\frac{4}{10} \cos \left(\frac{2 k \pi}{M}\right)-\mathbf{i} \frac{2}{10} \sin \left(\frac{2 k \pi}{M}\right), \\
& \lambda_{\mathbf{B}}^{(k)}=\frac{19}{30}+\frac{11}{30} \cos \left(\frac{2 k \pi}{M}\right)-\mathbf{i} \frac{29}{30} \sin \left(\frac{2 k \pi}{M}\right), \\
& k=0, \cdots M-1 .
\end{aligned}
$$

The eigenvalues of $\mathbf{C A}^{-\mathbf{1}} \mathbf{B}$ are

$$
\lambda_{\mathbf{C A}^{-1} \mathbf{B}}^{(k)}=\frac{\lambda_{\mathbf{C}}^{(k)} \lambda_{\mathbf{B}}^{(k)}}{\lambda_{\mathbf{A}}^{(k)}}, k=0, \cdots M-1 .
$$

We consider von Neumann stability analysis of the third-order SSP RungeKutta method (12) for the solution of the semidiscrete problem. The amplifier matrix can be deduced as follows

$$
G=1+\sigma \mathbf{S}+\frac{1}{2}(\sigma \mathbf{S})^{2}+\frac{1}{6}(\sigma \mathbf{S})^{3},
$$

where $\sigma=\lambda \Delta t / h, \mathbf{S}=-\mathbf{C A}^{-1} \mathbf{B}$. The eigenvalues of the matrix $\mathbf{S}$ satisfy

$$
\lambda_{\mathbf{G}}^{(k)}=1+\sigma \lambda_{\mathbf{S}}^{(k)}+\frac{1}{2}\left(\sigma \lambda_{\mathbf{S}}^{(k)}\right)^{2}+\frac{1}{6}\left(\sigma \lambda_{\mathbf{S}}^{(k)}\right)^{3}, k=0, \cdots M-1 .
$$

The stability condition for the present scheme with the fourth-order RungeKutta method is $\sigma<1.0$ [27] and third-order SSP Runge-Kutta method (12) is $\sigma<0.9$ [42]. 
The stability analysis of the diffusion equations for the Runge-Kutta method with sixth-order Padé finite volume scheme with periodic boundary conditions is discussed in [34].

A convergence study of the linear upwind compact (5) for smooth solutions was presented in [27]. The convergence properties of the nonlinear upwind scheme (7) is severely influenced by nonlinear weights. The convergence of the present scheme with different nonlinear weights for conservation law are discussed in [36]. The present nonlinear weighted compact scheme which is coupled the fifth-order upwind (7) for advection term with sixth-order Padé finite volume scheme for diffusion terms can converge at fifth-order.

\section{Numerical examples}

In this section, we provide some classical numerical examples with both smooth initial conditions and initial discontinuous distributions for the fifthorder finite volume compact WENO scheme with the third order SSP RungeKutta time discreization, which is denoted as the "FVCW" scheme. The original high order Padé finite volume methods scheme of Kobayashi [34] is denoted as "HOP". The discrete $L_{1}, L_{2}$ and $L_{\infty}$ error norms are defined as follows

$\left\|e_{n}\right\|_{L_{1}}=\frac{1}{N} \sum_{j=1}^{N}\left|\bar{u}_{j}-\tilde{u}_{j}\right|,|| e_{n}\left\|_{L_{\infty}}=\max _{1 \leq j \leq N}\left|\bar{u}_{j}-\tilde{u}_{j}\right|,|| e_{n}\right\|_{L_{2}}=\left(\sum_{j=1}^{N}\left|\bar{u}_{j}-\tilde{u}_{j}\right|^{2} \Delta x\right)^{\frac{1}{2}}$

where $\bar{u}$ and $\tilde{u}$ are exact and numerical solution respectively.

Example 1. In this example, we consider (1) with initial condition in the following form

$$
u(x, 0)=2 \nu \frac{\pi \sin (\pi x)}{\sigma+\cos (\pi x)}, x \in(0,1),
$$

and the boundary conditions

$$
u(0, t)=0, u(1, t)=0 .
$$

The exact solution of this problem is given in [43]

$$
u(x, t)=\frac{2 \nu \pi e^{-\pi^{2} \nu t} \sin (\pi x)}{\sigma+e^{-\pi^{2} \nu t} \cos (\pi x)} .
$$


The numerical error and numerical rates of convergence of the present finite volume compact-WENO scheme can be investigated with this closed form of the exact solution.

In Table 1, the numerical results for $\sigma=2 . R e=2,10$ are compared with those obtained in [12], [22] and [16]. We can observe that FVCW schemes have lower $L_{2}$ errors than other three schemes. We compare our numerical results for $\sigma=2$. $R e=10$ with those obtained in Liao [6] and classical fifth-order Padé finite volume methods in Table 2 and observe that the present scheme can obtain fifth-order accuracy and the $L_{2}$ errors are lower than that by implicit fourth-order finite-difference scheme [6]. The $L_{2}$ errors and orders for fifth-order Padé finite volume scheme, WENO scheme and the present FVCW scheme for $\sigma=2$. Re $=1000$ are given in Table 3 . We observe that the FVCW scheme can obtain fifth-order accuracy and the $L_{2}$ errors are lower than those obtained with WENO scheme. In table 4 , we compare our results with results of Mittal [16] and Jiwari [13] for $\sigma=200$ and $\Delta t=0.01$ at $T=1$. In this case, the fifth-order of accuracy can also be maintained and the $L_{\infty}$ and $L_{2}$ errors of present schemes are much lower than the errors of Jiwari [13] and Mittal [16].

Example 2. We consider (1) with the following initial condition

$$
u(x, 0)=\sin (\pi x), x \in(0,1),
$$

and the homogeneous boundary conditions

$$
u(0, t)=0, u(1, t)=0 .
$$

The exact solution for this example as given in [2]

$$
u(x, t)=2 \pi \nu \frac{\sum_{n=1}^{\infty} a_{n} \exp \left(-n^{2} \pi^{2} \nu t\right) n \sin (n \pi x)}{a_{0}+\sum_{n=1}^{\infty} a_{n} \exp \left(-n^{2} \pi^{2} \nu t\right) \cos (n \pi x)},
$$

with the Fourier coefficients are defined as

$$
\begin{gathered}
a_{0}=\int_{0}^{1} \exp \left\{-(2 \pi \nu)^{-1}[1-\cos (\pi x)]\right\} d x \\
a_{n}=2 \int_{0}^{1} \exp \left\{-(2 \pi \nu)^{-1}[1-\cos (\pi x)]\right\} \cos (n \pi x) d x, n=1,2,3, \cdots .
\end{gathered}
$$


Table 1: Comparison of results at $t=0.001, \sigma=2, \Delta t=0.0001, h=0.025$ for $\operatorname{Re}=2,10$.

\begin{tabular}{|c|c|c|c|c|c|c|}
\hline & & Exact & FVCW & Ganaie [12] & Asaithambi [22] & Mittal [16] \\
\hline \multirow{9}{*}{$\mathrm{Re}=2$} & 0.1 & 0.3278695524 & 0.3278695524 & 0.327871 & 0.327874 & 0.327870 \\
\hline & 0.2 & 0.6550692222 & 0.6550692220 & 0.655067 & 0.655078 & 0.655071 \\
\hline & 0.3 & 0.9784124992 & 0.9784124987 & 0.978418 & 0.978427 & 0.978416 \\
\hline & 0.4 & 1.2884634969 & 1.2884634950 & 1.288464 & 1.288485 & 1.288469 \\
\hline & 0.5 & 1.5630638524 & 1.5630638350 & 1.563061 & 1.563096 & 1.563074 \\
\hline & 0.6 & 1.7566421091 & 1.7566419249 & 1.756648 & 1.756691 & 1.756654 \\
\hline & 0.7 & 1.7872063975 & 1.7872065979 & 1.787201 & 1.787281 & 1.787204 \\
\hline & 0.8 & 1.5376943907 & 1.5376944007 & 1.537693 & 1.537794 & 1.537649 \\
\hline & 0.9 & 0.9168597988 & 0.9168597587 & 0.916880 & 0.916941 & 0.916786 \\
\hline \multirow{13}{*}{$\mathrm{Re}=10$} & $L_{\infty}$ error & & $8.36 \mathrm{E}-07$ & $2.00 \mathrm{E}-05$ & & $7.44 \mathrm{E}-05$ \\
\hline & $L_{2}$ error & & $2.05 \mathrm{E}-07$ & $3.54 \mathrm{E}-06$ & & $2.79 \mathrm{E}-05$ \\
\hline & 0.1 & 0.0657497591 & 0.0657497591 & 0.065750 & 0.065750 & 0.065750 \\
\hline & 0.2 & 0.1313829355 & 0.1313829355 & 0.131383 & 0.131383 & 0.131383 \\
\hline & 0.3 & 0.1962808678 & 0.1962808678 & 0.196281 & 0.196281 & 0.196281 \\
\hline & 0.4 & 0.2585757378 & 0.2585757375 & 0.258576 & 0.258576 & 0.258576 \\
\hline & 0.5 & 0.3138493556 & 0.3138493522 & 0.313848 & 0.313850 & 0.313850 \\
\hline & 0.6 & 0.3529718209 & 0.3529717855 & 0.352972 & 0.352972 & 0.352972 \\
\hline & 0.7 & 0.3594428596 & 0.3594429072 & 0.359443 & 0.359444 & 0.359443 \\
\hline & 0.8 & 0.3095803849 & 0.3095803878 & 0.309580 & 0.309583 & 0.309579 \\
\hline & 0.9 & 0.1847537428 & 0.1847537364 & 0.184752 & 0.184756 & 0.184751 \\
\hline & $L_{\infty}$ error & & $1.55 \mathrm{E}-07$ & $2.00 \mathrm{E}-06$ & & $3.08 \mathrm{E}-06$ \\
\hline & $L_{2}$ error & & 3.60E-08 & $3.54 \mathrm{E}-07$ & & $1.15 \mathrm{E}-06$ \\
\hline
\end{tabular}

Table 2: Numerical errors and convergence rate for Example 1 with $\sigma=2, R e=10$, $T=1$.

\begin{tabular}{llllllll}
\hline & \multicolumn{2}{l}{ Liao [6], $\Delta t=0.000001$} & \multicolumn{2}{l}{$\mathrm{FVCW}, \Delta t=C \Delta x^{2} / R e$} & \multicolumn{2}{l}{ HOP, $\Delta t=C \Delta x^{2} / R e$} \\
\hline $\mathrm{N}$ & $L_{\infty}$ error & $L_{\infty}$ order & $L_{\infty}$ error & $L_{\infty}$ order & $L_{\infty}$ error & $L_{\infty}$ order \\
\hline 10 & $1.5763 \mathrm{E}-05$ & - & $1.033 \mathrm{E}-05$ & - & $8.094 \mathrm{E}-06$ & - \\
20 & $9.8231 \mathrm{E}-07$ & 4.004 & $3.805 \mathrm{E}-07$ & 4.76 & $3.817 \mathrm{E}-07$ & 4.41 \\
40 & $6.1273 \mathrm{E}-08$ & 4.003 & $1.273 \mathrm{E}-08$ & 4.90 & $1.272 \mathrm{E}-08$ & 4.91 \\
80 & $3.8303 \mathrm{E}-09$ & 4.000 & $4.033 \mathrm{E}-10$ & 4.98 & $4.015 \mathrm{E}-10$ & 4.99 \\
160 & $2.3954 \mathrm{E}-10$ & 3.999 & $1.262 \mathrm{E}-11$ & 5.00 & $1.269 \mathrm{E}-11$ & 4.98 \\
\hline
\end{tabular}

Table 3: Numerical errors and convergence rate for Example 1 with $\sigma=2, R e=1000, \mathrm{~T}=1$

\begin{tabular}{lllllll}
\hline & \multicolumn{2}{l}{$\mathrm{HOP}, \Delta t=C \Delta x^{2} / R e$} & \multicolumn{2}{l}{ WENO, $\Delta t=C \Delta x^{2} / R e$} & \multicolumn{2}{l}{$\mathrm{FVCW}, \Delta t=C \Delta x^{2} / R e$} \\
\hline $\mathrm{N}$ & $L_{2}$ error & $L_{2}$ order & $L_{2}$ error & $L_{2}$ order & $L_{2}$ error & $L_{2}$ order \\
\hline 20 & $5.451 \mathrm{E}-09$ & - & $2.131 \mathrm{E}-07$ & - & $6.439 \mathrm{E}-08$ & - \\
40 & $2.508 \mathrm{E}-10$ & 4.44 & $6.673 \mathrm{E}-09$ & 5.00 & $6.055 \mathrm{E}-10$ & 6.73 \\
80 & $8.456 \mathrm{E}-12$ & 4.89 & $1.787 \mathrm{E}-10$ & 5.22 & $1.174 \mathrm{E}-11$ & 5.69 \\
160 & $2.687 \mathrm{E}-13$ & 4.98 & $5.158 \mathrm{E}-12$ & 5.11 & $2.782 \mathrm{E}-13$ & 5.40 \\
320 & $8.664 \mathrm{E}-15$ & 4.95 & $1.564 \mathrm{E}-13$ & 5.04 & $8.693 \mathrm{E}-15$ & 5.00 \\
\hline
\end{tabular}


Table 4: Numerical errors and convergence rate for Example 1 with $\sigma=100, R e=200$, $T=1$.

\begin{tabular}{cccccccccc}
\hline & \multicolumn{3}{c}{ Mittal $[16], \Delta t=0.01$} & \multicolumn{1}{c}{ Jiwari $[13], \Delta t=0.01$} & \multicolumn{5}{c}{ FVCW, $\Delta t=C \Delta x^{2} / R e$} \\
\hline $\mathrm{N}$ & $L_{\infty}$ error & $L_{2}$ error & $L_{\infty}$ error & $L_{2}$ error & $L_{2}$ order & $L_{\infty}$ error & $L_{\infty}$ order & $L_{2}$ error & $L_{2}$ order \\
\hline 10 & $1.215 \mathrm{E}-07$ & $8.631 \mathrm{E}-08$ & $4.708 \mathrm{E}-08$ & $6.459 \mathrm{E}-08$ & - & $2.010 \mathrm{E}-09$ & - & $8.653 \mathrm{E}-10$ & - \\
20 & $3.062 \mathrm{E}-08$ & $2.153 \mathrm{E}-08$ & $1.091 \mathrm{E}-08$ & $4.465 \mathrm{E}-09$ & 2.11 & $7.071 \mathrm{E}-11$ & 4.83 & $2.019 \mathrm{E}-11$ & 5.42 \\
40 & $7.644 \mathrm{E}-09$ & $5.378 \mathrm{E}-09$ & $1.980 \mathrm{E}-09$ & $2.786 \mathrm{E}-10$ & 2.46 & $2.629 \mathrm{E}-12$ & 4.75 & $6.460 \mathrm{E}-13$ & 4.97 \\
80 & $1.917 \mathrm{E}-09$ & $1.345 \mathrm{E}-09$ & $7.182 \mathrm{E}-09$ & $2.665 \mathrm{E}-10$ & 1.86 & $8.941 \mathrm{E}-14$ & 4.88 & $2.047 \mathrm{E}-14$ & 4.98 \\
\hline
\end{tabular}

Numerical results obtained by the present finite volume compact-WENO method and the exact solution for (1) at time $T=0.1$ for $R e=1$ and $h=0.1,0.0125, \Delta t=0.0001,0.00001$ are shown in Table 5. We compare our numerical results with those obtained with sixth-order compact finite difference scheme [9], implicit fourth-order compact finite difference scheme [6] and local discontinuous Galerkin (LDG) finite element method [21]. These comparisons show that the present finite volume compact-WENO scheme obtains much better results than linear compact schemes [6] and LDG method [21]. The numerical results are almost the same as [9] with $h=0.1$, however the time step size we set $\Delta t=0.001$ in our computation while $\Delta t=0.00001$ in [9]. The numerical solutions with $R e=100$ at different times at different points are shown in Table 6 . Numerical results show that the present numerical solutions are in good agreement with the exact solutions. Table 6 also shows that the numerical solutions using the present scheme with $h=0.0125$ are much more accurate than the numerical solutions using automatic differentiation method [22]. The numerical results obtained by the present scheme with $h=0.025, \Delta t=0.001$ are slightly better than the results using fourth-order finite difference method with $h=0.0125, \Delta t=0.001$ [5]. The numerical solutions produced by the present scheme are much more accurate than the results using the modified cubic B-splines collocation method with $h=0.025, \Delta t=0.0025[16]$. Figure 1 shows the numerical solutions for $R e=1.0,10.0,100.0,10000.0$ at different times which exhibit the correct physical behavior of the problem. The figure also shows that the propagation front grow steeper as we set the Reynolds number larger. For $R e=20000.0$, the advection term dominates over the dissipation term. From Figure 2, we can observe that the classical linear fifth-order Padé finite volume yields oscillatory solutions across discontinuities, however, the FVCW scheme captures non-oscillatory solutions. 
Table 5: Comparison between exact and numerical solutions of Example 2 for $R e=1$ and $T=0.1$

\begin{tabular}{lllllll}
\hline $\mathrm{x}$ & FVCW & & Sari $[9]$ & Liao $[6]$ & Shao $[21]$ & Exact \\
& $h=0.1$ & $h=0.0125$ & $h=0.1$ & $h=0.0125$ & $h=0.1, k=4$ & \\
& $\Delta t=0.001$ & $\Delta t=0.00001$ & $\Delta t=0.00001$ & $\Delta t=0.00001$ & $\Delta t=0.00001$ & \\
\hline 0.1 & 0.1095410340 & 0.1095381513 & 0.10954 & 0.10953815119 & 0.10953815187843 & 0.1095381513 \\
0.2 & 0.2097937393 & 0.2097921489 & 0.20979 & 0.20979214867 & 0.20979215073044 & 0.2097921489 \\
0.3 & 0.2918993650 & 0.2918963509 & 0.29190 & 0.29189635032 & 0.29189635398773 & 0.2918963508 \\
0.4 & 0.3479248166 & 0.3479239124 & 0.34792 & 0.34792391150 & 0.34792391648131 & 0.3479239124 \\
0.5 & 0.3715814171 & 0.3715774762 & 0.37158 & 0.37157747490 & 0.37157748046658 & 0.3715774761 \\
0.6 & 0.3590491636 & 0.3590455801 & 0.35905 & 0.35904557846 & 0.35904558368977 & 0.3590455800 \\
0.7 & 0.3099099916 & 0.3099050008 & 0.30991 & 0.30990499905 & 0.30990500314125 & 0.3099050006 \\
0.8 & 0.2278224940 & 0.2278174068 & 0.22782 & 0.22781740532 & 0.22781740781517 & 0.2278174066 \\
0.9 & 0.1206949990 & 0.1206866913 & 0.12069 & 0.12068669034 & 0.12068669132062 & 0.1206866911 \\
\hline
\end{tabular}

Table 6: Comparison between exact and numerical solutions of Example 2 for $R e=100$.

\begin{tabular}{|c|c|c|c|c|c|c|c|}
\hline $\mathrm{x}$ & $\mathrm{T}$ & $\begin{array}{l}\text { FVCW } \\
h=0.025 \\
\Delta t=0.005\end{array}$ & $\begin{array}{l}h=0.0125 \\
\Delta t=0.0001\end{array}$ & $\begin{array}{l}\text { Hassanien [5] } \\
h=0.0125 \\
\Delta t=0.001\end{array}$ & $\begin{array}{l}\text { Mittal [16] } \\
h=0.01 \\
\Delta t=0.002\end{array}$ & $\begin{array}{l}\text { Asaithambi [22] } \\
h=0.0125 \\
\Delta t=0.0001\end{array}$ & Exact \\
\hline \multirow[t]{5}{*}{0.25} & 0.4 & 0.3419148998 & 0.3419149324 & 0.34191 & 0.34192 & 0.34193 & 0.3419149324 \\
\hline & 0.6 & 0.2689648148 & 0.2689648453 & 0.26896 & 0.26896 & 0.26898 & 0.2689648453 \\
\hline & 0.8 & 0.2214818903 & 0.2214819145 & 0.22148 & 0.22148 & 0.22149 & 0.2214819145 \\
\hline & 1.0 & 0.1881939425 & 0.1881939614 & 0.18819 & 0.18819 & 0.18820 & 0.1881939614 \\
\hline & 3.0 & 0.0751140806 & 0.0751140839 & 0.07511 & 0.07511 & 0.07512 & 0.0751140839 \\
\hline \multirow[t]{5}{*}{0.50} & 0.4 & 0.6607111017 & 0.6607109708 & 0.66071 & 0.66071 & 0.66076 & 0.6607109710 \\
\hline & 0.6 & 0.5294183037 & 0.5294182636 & 0.52942 & 0.52942 & 0.52945 & 0.5294182637 \\
\hline & 0.8 & 0.4391382575 & 0.4391382506 & 0.43914 & 0.43914 & 0.43916 & 0.4391382507 \\
\hline & 1.0 & 0.3744200327 & 0.3744200376 & 0.37442 & 0.37442 & 0.37443 & 0.3744200376 \\
\hline & 3.0 & 0.1501789931 & 0.1501790052 & 0.15218 & 0.15018 & 0.15018 & 0.1501790052 \\
\hline \multirow[t]{5}{*}{0.75} & 0.4 & 0.9102646129 & 0.9102645487 & 0.91027 & 0.91027 & 0.91046 & 0.9102645462 \\
\hline & 0.6 & 0.7672501164 & 0.7672432886 & 0.76724 & 0.76724 & 0.76733 & 0.7672432826 \\
\hline & 0.8 & 0.6474031323 & 0.6473952374 & 0.64740 & 0.64740 & 0.64744 & 0.6473952348 \\
\hline & 1.0 & 0.5560569314 & 0.5560506935 & 0.55605 & 0.55605 & 0.55608 & 0.5560507045 \\
\hline & 3.0 & 0.2248087625 & 0.2248112673 & 0.22481 & 0.22483 & 0.22484 & 0.2248112482 \\
\hline
\end{tabular}




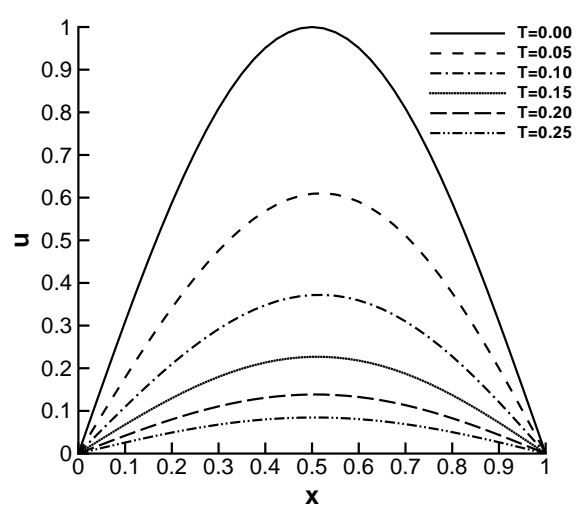

(a) $\mathrm{Re}=1, \Delta t=0.001, h=0.025$

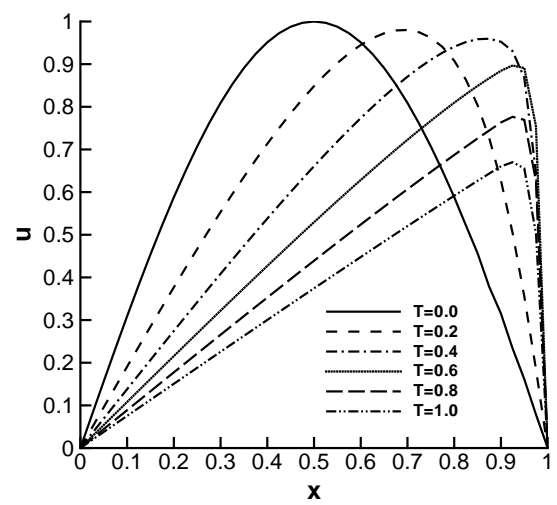

(c) $\operatorname{Re}=100, \Delta t=0.01, h=0.025$

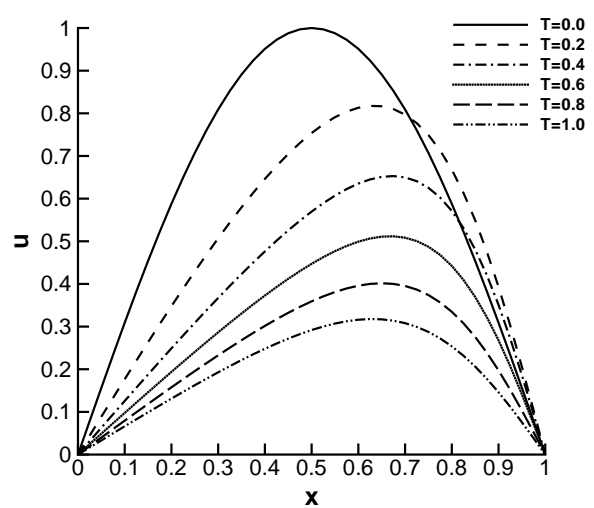

(b) $\operatorname{Re}=10, \Delta t=0.01, h=0.025$

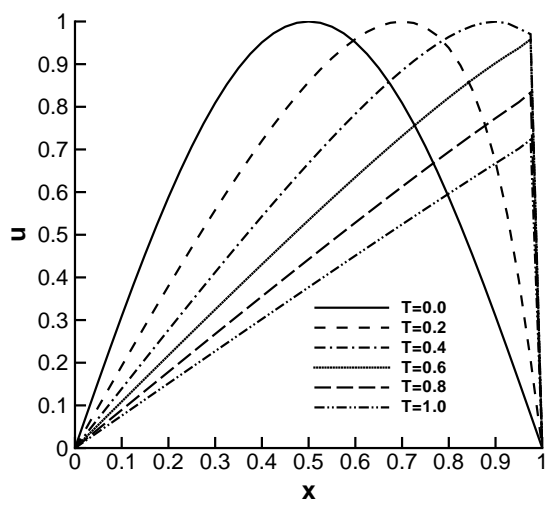

(d) $\operatorname{Re}=10000, \Delta t=0.01, h=0.025$

Figure 1: Numerical solutions for Example 2 


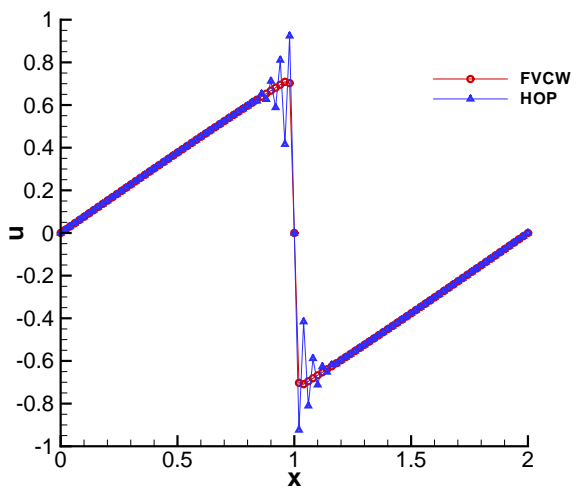

(a) $\operatorname{Re}=20000, T=0.6$

Figure 2: Numerical solutions for Example 2

Example 3. The initial condition for this example is

$$
u(x, 0)=4 x(1-x), x \in(0,1),
$$

and the boundary conditions are

$$
u(0, t)=0, u(1, t)=0 .
$$

The exact solution of this example is also given by Eq.(5), but in this case the Fourier coefficients are

$$
\begin{gathered}
a_{0}=\int_{0}^{1} \exp \left(-\frac{-3 x^{2}-2 x^{3}}{3 \nu}\right) d x \\
a_{n}=\int_{0}^{1} \exp \left(-\frac{-3 x^{2}-2 x^{3}}{3 \nu}\right) \cos (n \pi x) d x, n=1,2,3, \cdots .
\end{gathered}
$$$$
\text { Table } 7 \text { shows that computed solutions for this example with } R e=100 \text { are }
$$
in good agreement with exact solutions. The numerical solutions obtained by using the present compact-WENO scheme are compared with those obtained by using compact finite difference scheme $[6,8]$ and modified cubic B-splines collocation method [16] in Table 7 . The numerical comparisons show that the present scheme produces better results than Liao [6] and Mittal [16]. 
Table 7: Comparison between exact and numerical solutions of Example 3 for $R e=100$.

\begin{tabular}{lllllll}
\hline $\mathrm{x}$ & T & FVCW & Liao [6] & Zhang [8] & Mittal [16] & Exact \\
& & $h=0.025$ & $h=0.0125$ & $h=0.025$ & $h=0.025$ & \\
& & $\Delta t=0.001$ & $\Delta t=0.01$ & $\Delta t=0.001$ & $\Delta t=0.001$ & \\
\hline 0.25 & 0.4 & 0.36226 & 0.36226 & 0.36226 & 0.36225 & 0.36226 \\
& 0.6 & 0.28204 & 0.28204 & 0.28203 & 0.28202 & 0.28204 \\
& 0.8 & 0.23045 & 0.23045 & - & 0.23044 & 0.23045 \\
& 1.0 & 0.19469 & 0.19469 & 0.19469 & 0.19468 & 0.19469 \\
& 3.0 & 0.07613 & 0.07606 & 0.07613 & 0.07613 & 0.07613 \\
0.50 & 0.4 & 0.68368 & 0.68369 & 0.68367 & 0.68368 & 0.68368 \\
& 0.6 & 0.54832 & 0.54832 & 0.54831 & 0.54832 & 0.54832 \\
& 0.8 & 0.45371 & 0.45372 & - & 0.45371 & 0.45371 \\
& 1.0 & 0.38568 & 0.38568 & 0.38567 & 0.38567 & 0.38568 \\
& 3.0 & 0.15218 & 0.15203 & 0.15218 & 0.15218 & 0.15218 \\
0.75 & 0.4 & 0.92050 & 0.92040 & 0.92051 & 0.92052 & 0.92050 \\
& 0.6 & 0.78300 & 0.78304 & 0.78301 & 0.78300 & 0.78299 \\
& 0.8 & 0.66273 & 0.66276 & - & 0.66272 & 0.66272 \\
& 1.0 & 0.56933 & 0.56934 & 0.56932 & 0.56932 & 0.56932 \\
& 3.0 & 0.22774 & 0.22751 & 0.22774 & 0.22782 & 0.22774 \\
\hline
\end{tabular}

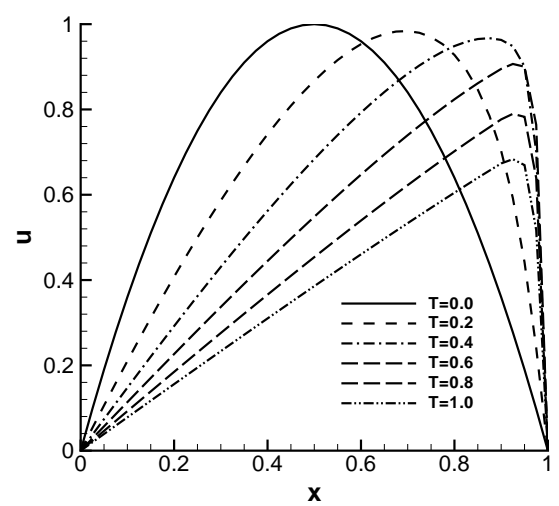

Figure 3: Numerical solutions for Example 3, $\mathrm{Re}=100, \Delta t=0.001, h=0.025$ 
Table 8: Comparison between exact and numerical solutions of Example 4 for $R e=200$.

\begin{tabular}{lllllllll}
\hline \multicolumn{3}{l}{ Korkmaz $[24]$} & \multicolumn{2}{l}{ Haq $[23]$} & \multicolumn{2}{l}{ FVCW } \\
\multicolumn{2}{l}{$h=0.02, \Delta t=0.01$} & \multicolumn{2}{l}{$h=0.01, \Delta t=0.001$} & \multicolumn{2}{l}{$h=0.02, \Delta t=0.01$} & \multicolumn{2}{l}{$h=0.01, \Delta t=0.001$} \\
\hline $\mathrm{T}$ & $L_{\infty}$ & $L_{2}$ & $L_{\infty}$ & $L_{2}$ & $L_{\infty}$ & $L_{2}$ & $L_{\infty}$ & $L_{2}$ \\
\hline 1.7 & $2.3 \mathrm{E}-05$ & $1.0 \mathrm{E}-05$ & $5.6554 \mathrm{E}-04$ & $9.51 \mathrm{E}-06$ & $5.929 \mathrm{E}-05$ & $1.173 \mathrm{E}-05$ & $1.623 \mathrm{E}-06$ & $2.976 \mathrm{E}-07$ \\
2.4 & $3.5 \mathrm{E}-05$ & $1.3 \mathrm{E}-05$ & $3.3077 \mathrm{E}-04$ & $2.96 \mathrm{E}-06$ & $2.199 \mathrm{E}-05$ & $4.518 \mathrm{E}-06$ & $5.072 \mathrm{E}-07$ & $1.114 \mathrm{E}-07$ \\
3.1 & $4.2 \mathrm{E}-04$ & $4.8 \mathrm{E}-03$ & $1.7990 \mathrm{E}-05$ & $1.951 \mathrm{E}-05$ & $7.19 \mathrm{E}-06$ & $2.073 \mathrm{E}-06$ & $4.830 \mathrm{E}-06$ & $6.028 \mathrm{E}-07$ \\
\hline
\end{tabular}

Example 4. In this example, we consider the Shock-like solution of (1), and the analytical solution given in [44] is

$$
u(x, t)=\frac{\frac{x}{t}}{1+\sqrt{\frac{t}{t_{0}}} \exp \left(\frac{x^{2}}{4 \nu t}\right)}, t \geq 1,
$$

where $t_{0}=\exp \left(\frac{1}{8 \nu}\right)$. Initial condition is obtained from the analytical solution when $t=1$ is used. Boundary conditions are set to be $u(a, t)=u(b, t)=0$.

In our computation, we set $R e=200,2000, a=0, b=1, \Delta t=0.01,0.001$ and $h=0.02,0.01$. The $L_{\infty}$ and $L_{2}$ errors at various times are compared with results using differential quadrature method [24] and meshless method of lines [23]. Table 8 shows that the present scheme produces better results than [24] at times $T=2.4,3.1$. However, the results at time $T=1.7$ obtained in [24] are slightly better than that using the present scheme. It is evident from Table 8 that the present compact-WENO scheme gives much better results as compared to meshless method of lines [23]. The numerical results with $R e=200$ and $R e=2000$ at various times are depicted in Figure 4 . It can be seen that for the smaller viscosity value the propagation front is steeper.

Example 5. The initial and boundary conditions for this example are

$$
u(x, 0)= \begin{cases}1, & \text { if } 0 \leq x<5 \\ 6-x, & \text { if } 5 \leq x<6 \\ 0, & \text { if } 6 \leq x<12\end{cases}
$$

and $u(0, t)=1, u(12, t)=0$.

The numerical solutions of this example have been studied by Mittal [16], Asaithambi [22] as an example of a shock wave approaching steady state. 


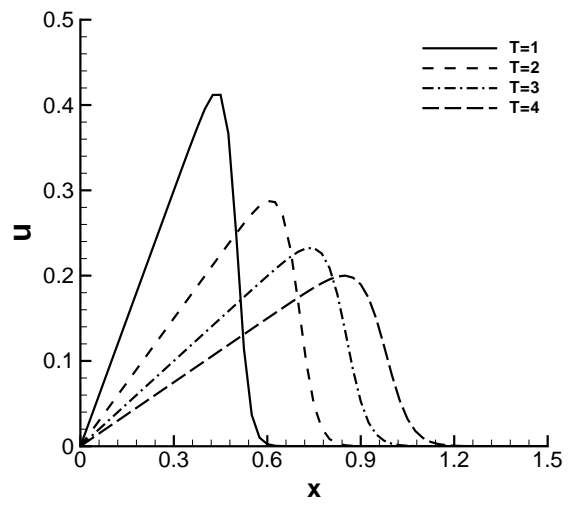

(a) $\operatorname{Re}=200, \Delta t=0.01, h=0.02$

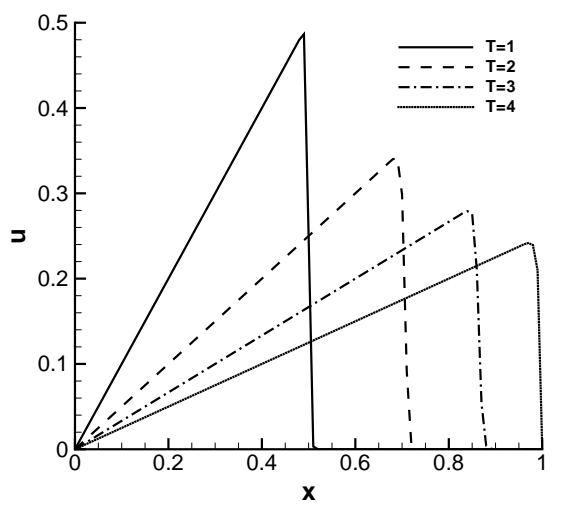

(b) $\operatorname{Re}=2000, \Delta t=0.001, h=0.01$

Figure 4: Numerical solutions for Example 4

Figure 5 shows that correct physical behavior for different values of Reynolds number can be captured by the present method with $h=0.1$. Figure 5 also shows that the propagation front grow steeper as we set the Reynolds number larger.

Example 6. As the last test example we consider the particular solution of (1) as given in [45]

$$
u(x, t)=\frac{\alpha+\mu+(\mu-\alpha) \exp \eta}{1+\exp \eta}, 0 \leq x \leq 1, t \geq 0,
$$

where $\eta=\frac{\alpha(x-\mu t-\gamma)}{\nu}$, and $\alpha, \mu$ and $\gamma$ are chosen as $\alpha=0.4, \mu=0.6$ and $\gamma=0.125$. The initial condition is selected as the analytic solution at time $t=0$. The boundary conditions are chosen as

$$
u(0, t)=1, u(1, t)=0.2 .
$$

To compare with Xu [11], Dağ [10] and Mittal [16], simulations are carried out using the parameters $R e=100, h=1 / 36$ and $\Delta t=0.001$, and numerical results are shown in Table 9. It can be seen that the numerical results obtained by the present compact-WENO scheme are much better than the results in [11], [10] and [16]. Numerical solutions for $R e=100$ at different times which exhibit the correct physical behavior of the problem are shown in Figure 6. 


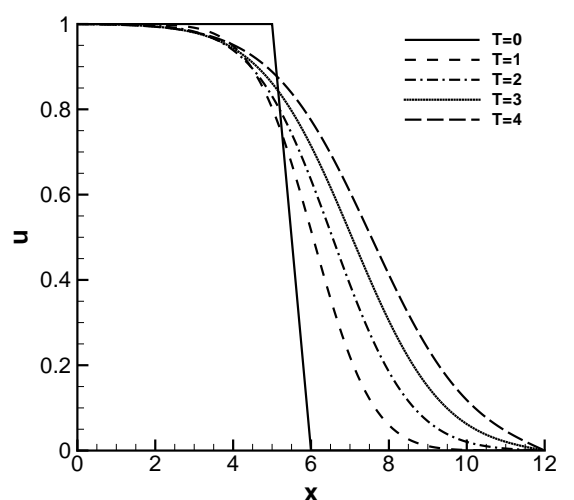

(a) $\operatorname{Re}=1, \Delta t=0.001$

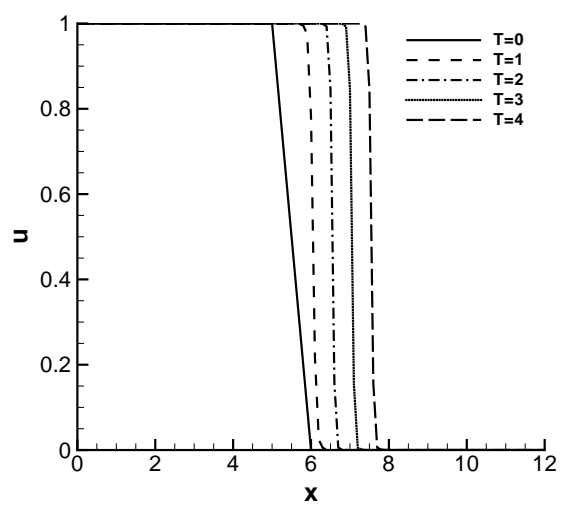

(c) $\operatorname{Re}=100, \Delta t=0.01$

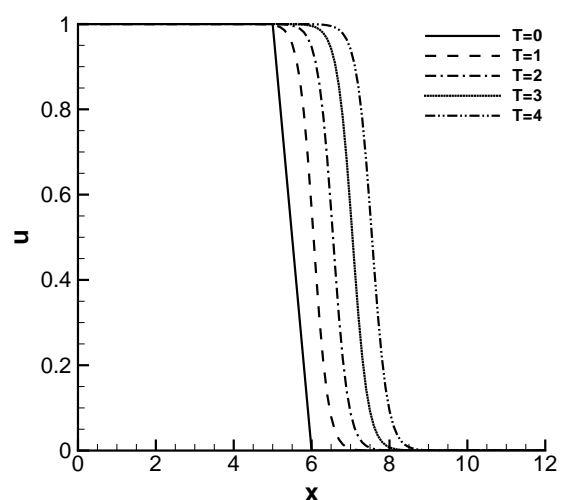

(b) $\operatorname{Re}=10, \Delta t=0.01$

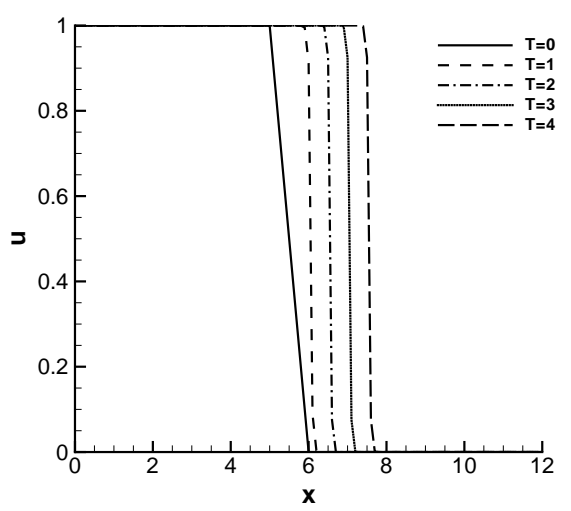

(d) $\operatorname{Re}=1000, \Delta t=0.01$

Figure 5: Numerical solutions for Example 5 with $h=0.1$ 
Table 9: Comparison between exact and numerical solutions of Example 6 at $T=0.5$ for $R e=100$.

\begin{tabular}{llllll}
\hline $\mathrm{x}$ & FVCW & $\mathrm{Xu}[11]$ & Dağ [10] & Mittal [16] & Exact \\
& $h=1 / 36$ & $h=1 / 36$ & $h=1 / 36$ & $h=1 / 36$ & \\
& $\Delta t=0.01$ & $\Delta t=0.001$ & $\Delta t=0.025$ & $\Delta t=0.01$ & \\
\hline 0.000 & 1.000 & 1.000 & 1.000 & 1.000 & 1.000 \\
0.056 & 1.000 & 1.000 & 1.000 & 1.000 & 1.000 \\
0.111 & 1.000 & 1.000 & 1.000 & 1.000 & 1.000 \\
0.167 & 1.000 & 1.000 & 1.000 & 1.000 & 1.000 \\
0.222 & 1.000 & 1.000 & 1.000 & 1.000 & 1.000 \\
0.278 & 0.998 & 0.998 & 0.999 & 0.998 & 0.998 \\
0.333 & 0.980 & 0.980 & 0.986 & 0.982 & 0.980 \\
0.389 & 0.848 & 0.859 & 0.850 & 0.844 & 0.847 \\
0.444 & 0.454 & 0.451 & 0.448 & 0.458 & 0.452 \\
0.500 & 0.238 & 0.237 & 0.236 & 0.238 & 0.238 \\
0.556 & 0.204 & 0.204 & 0.204 & 0.203 & 0.204 \\
0.611 & 0.200 & 0.200 & 0.200 & 0.200 & 0.200 \\
0.667 & 0.200 & 0.200 & 0.200 & 0.200 & 0.200 \\
0.722 & 0.200 & 0.200 & 0.200 & 0.200 & 0.200 \\
0.778 & 0.200 & 0.200 & 0.200 & 0.200 & 0.200 \\
0.833 & 0.200 & 0.200 & 0.200 & 0.200 & 0.200 \\
0.889 & 0.200 & 0.200 & 0.200 & 0.200 & 0.200 \\
0.944 & 0.200 & 0.200 & 0.200 & 0.200 & 0.200 \\
1.000 & 0.200 & 0.200 & 0.200 & 0.200 & 0.200 \\
$L_{1}$ error & $1.897 \mathrm{E}-04$ & $1.2 \mathrm{E}-03$ & $8.3 \mathrm{E}-03$ & $5.97 \mathrm{E}-03$ & \\
$L_{2}$ error & $4.655 \mathrm{E}-04$ & $2.9 \mathrm{E}-03$ & $1.2 \mathrm{E}-03$ & $1.45 \mathrm{E}-03$ & \\
\hline & & & & & \\
\hline
\end{tabular}

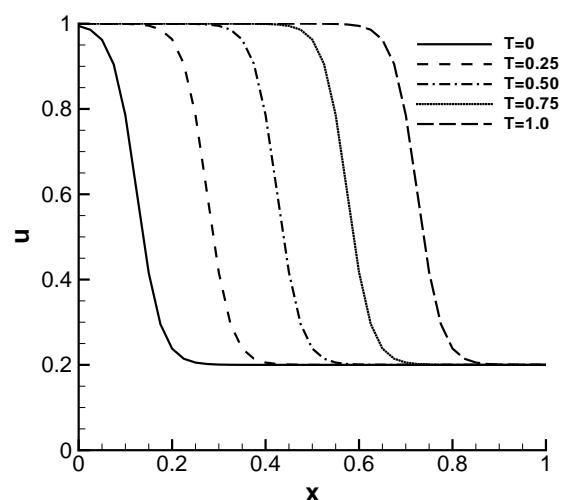

Figure 6: Numerical solutions for Example 6, $\mathrm{Re}=100, \Delta t=0.001, h=0.025$ 


\section{Conclusions}

In this paper, we develop a high-order finite volume compact scheme in space with a third-order SSP Runge-Kutta scheme in time for solving nonlinear Burgers' equation. In the present scheme, advective terms are computed by the fifth-order finite volume upwind compact-WENO scheme, while the diffusive terms are discretized by using the six-order Padé finite volume methods. Comparing with classical WENO schemes, the proposed compact schemes have spectral like resolution properties by using the global grid. We need to calculate the nonlinear weights at each time stage, then the disadvantage of high computational cost for compact schemes becomes even worse for the present nonlinear compact-WENO scheme. To achieve the essentially non-oscillatory properties of the present compact scheme, lower order compact stencils are combined with the noninear WENO weights to yield a fifth-order upwind compact interpolation. Some examples are tested, the numerical results show that the present scheme has better resolution compared to the classical finite volume non-compact WENO scheme. The present scheme can also produce essentially non-oscillatory solutions compared to the classical finite volume compact scheme. The numerical results show the good performance and high resolution property of the proposed FVCW scheme. The application of the scheme on non-uniform grids will be studied in our future work.

\section{Acknowledgement}

The work was partly supported by the Fundamental Research Funds for the Central Universities(2012QNB07, 2015QNA46).

\section{References}

[1] E. Hopf, The partial differential equation $u_{t}+u u_{x}=\mu u_{x x}$, Comm. Pure Appl. Math. 3 (1950) 201-230.

[2] J. D. Cole, et al., On a quasilinear parabolic equations occurring in aerodynamics, Quart. Appl. Math 9 (1951) 225-236.

[3] E. R. Benton, G. W. Platzman, A table of solutions of the onedimensional Burgers' equations, Quart. Appl. Math 30 (1972) 195-212. 
[4] S. Dhawan, S. Kapoor, S. Kumar, S. Rawat, Contemporary review of techniques for the solution of nonlinear Burgers equation, J. Comput. Sci. 3 (2012) 405-419.

[5] I. Hassanien, A. Salama, H. Hosham, Fourth-order finite difference method for solving Burgers' equation, Appl. Math. Comput. 170 (2005) 781-800.

[6] W. Liao, An implicit fourth-order compact finite difference scheme for one-dimensional Burgers' equation, Appl. Math. Comput. 206 (2008) $755-764$

[7] M. Gülsu, A finite difference approach for solution of Burgers' equation, Appl. Math. Comput. 175 (2006) 1245-1255.

[8] P.-G. Zhang, J.-P. Wang, A predictor-corrector compact finite difference scheme for Burgers' equation, Appl. Math. Comput. 219 (2012) 892-898.

[9] M. Sari, G. Gürarslan, A sixth-order compact finite difference scheme to the numerical solutions of Burgers' equation, Appl. Math. Comput. 208 (2009) 475-483.

[10] İ. Dağ, D. Irk, B. Saka, A numerical solution of the Burgers' equation using cubic B-splines, Appl. Math. Comput. 163 (2005) 199-211.

[11] M. Xu, R.-H. Wang, J.-H. Zhang, Q. Fang, A novel numerical scheme for solving Burgers' equation, Appl. Math. Comput. 217 (2011) 4473-4482.

[12] I. Ganaie, V. Kukreja, Numerical solution of Burgers' equation by cubic Hermite collocation method, Applied Mathematics and Computation 237 (2014) 571-581.

[13] R. Jiwari, R. Mittal, K. K. Sharma, A numerical scheme based on weighted average differential quadrature method for the numerical solution of Burgers' equation, Appl. Math. Comput. 219 (2013) 6680-6691.

[14] R. Jiwari, A haar wavelet quasilinearization approach for numerical simulation of Burgers' equation, Computer Physics Communications 183 (2012) 2413-2423. 
[15] R. Jiwari, A hybrid numerical scheme for the numerical solution of the Burgers' equation, Computer Physics Communications 188 (2015) $59-67$.

[16] R. Mittal, R. Jain, Numerical solutions of nonlinear burgers equation with modified cubic b-splines collocation method, Applied Mathematics and Computation 218 (2012) 7839-7855.

[17] R. Mittal, R. Jiwari, K. K. Sharma, A numerical scheme based on differential quadrature method to solve time dependent Burgers' equation, Engineering Computations 30 (2012) 117-131.

[18] R. Mittal, R. Jiwari, A differential quadrature method for numerical solutions of Burgers'-type equations, International Journal of Numerical Methods for Heat \& Fluid Flow 22 (2012) 880-895.

[19] V. Mukundan, A. Awasthi, Efficient numerical techniques for Burgers' equation, Applied Mathematics and Computation 262 (2015) 282-297.

[20] M. Kumar, S. Pandit, A composite numerical scheme for the numerical simulation of coupled Burgers' equation, Computer Physics Communications 185 (2014) 809-817.

[21] L. Shao, X. Feng, Y. He, The local discontinuous Galerkin finite element method for Burgers' equation, Math. Comput. Model. 54 (2011) 29432954.

[22] A. Asaithambi, Numerical solution of the Burgers' equation by automatic differentiation, Appl. Math. Comput. 216 (2010) 2700-2708.

[23] S. Haq, A. Hussain, M. Uddin, On the numerical solution of nonlinear Burgers'-type equations using meshless method of lines, Applied Mathematics and Computation 218 (2012) 6280-6290.

[24] A. Korkmaz, I. Dag, Polynomial based differential quadrature method for numerical solution of nonlinear Burgers' equation, J. Franklin Inst. 348 (2011) 2863-2875.

[25] S. Lele, Compact finite difference schemes with spectral-like resolution, J. Comput. Phys. 103 (1992) 16-42. 
[26] C.-W. Shu, High order weighted essentially nonoscillatory schemes for convection dominated problems, SIAM review 51 (2009) 82-126.

[27] S. Pirozzoli, Conservative hybrid compact-WENO schemes for shockturbulence interaction, J. Comput. Phys. 178 (2002) 81-117.

[28] X. Liu, S. Zhang, H. Zhang, C.-W. Shu, A new class of central compact schemes with spectral-like resolution I: Linear schemes, Journal of Computational Physics 248 (2013) 235-256.

[29] X. Liu, S. Zhang, H. Zhang, C.-W. Shu, A new class of central compact schemes with spectral-like resolution II: Hybrid weighted nonlinear schemes, Journal of Computational Physics 284 (2015) 133-154.

[30] X. Deng, H. Maekawa, Compact high-order accurate nonlinear schemes, J. Comput. Phys. 130 (1997) 77-91.

[31] X. Deng, H. Zhang, Developing high-order weighted compact nonlinear schemes, J. Comput. Phys. 165 (2000) 22-44.

[32] L. Jiang, H. Shan, C. Liu, Weighted compact scheme for shock capturing, Int. J. Comput. Fluid D. 15 (2001) 147-155.

[33] D. Ghosh, J. Baeder, Compact reconstruction schemes with weighted ENO limiting for hyperbolic conservation laws, SIAM. J. Sci. Comput. 34 (2012) 1678-1706.

[34] M. Kobayashi, On a class of Padé finite volume methods, J. Comput. Phys. 156 (1999) 137-180.

[35] M. Piller, E. Stalio, Compact finite volume schemes on boundary-fitted grids, J. Comput. Phys. 227 (2008) 4736-4762.

[36] Y. Guo, T. Xiong, Y. Shi, A maximum-principle-satisfying high-order finite volume compact WENO scheme for scalar conservation laws with applications in incompressible flows, Journal of Scientific Computing (2014) 1-27.

[37] Y. Guo, T. Xiong, Y. Shi, A positivity-preserving high order finite volume compact-WENO scheme for compressible Euler equations, J. Comput. Phys. 274 (2014) 505-523. 
[38] R. Borges, M. Carmona, B. Costa, W. Don, An improved weighted essentially non-oscillatory scheme for hyperbolic conservation laws, J. Comput. Phys. 227 (2008) 3191-3211.

[39] M. Castro, B. Costa, W. Don, High order weighted essentially nonoscillatory WENO-Z schemes for hyperbolic conservation laws, J. Comput. Phys. 230 (2011) 1766-1792.

[40] G.-S. Jiang, C.-W. Shu, Efficient implementation of weighted ENO schemes, J. Comput. Phys. 126 (1996) 202-228.

[41] H. Lomax, T. H. Pulliam, D. W. Zingg, Fundamentals of computational fluid dynamics, Springer Science \& Business Media, 2013.

[42] D. GHOSH, E. M. CONSTANTINESCU, J. BROWN, Efficient implementation of nonlinear compact schemes on massively parallel platforms, Submitted (????).

[43] W. Wood, An exact solution for Burgers' equation, Commun. Numer. Methods Eng. 22 (2006) 797-798.

[44] S. Harris, Sonic shocks governed by the modified Burgers' equation, EJAM 7 (1996) 201.

[45] I. Christie, D. F. Griffiths, A. R. Mitchell, J. M. Sanz-Serna, Product approximation for non-linear problems in the finite element method, IMA, J. Num. Anal. 1 (1981) 253-266. 